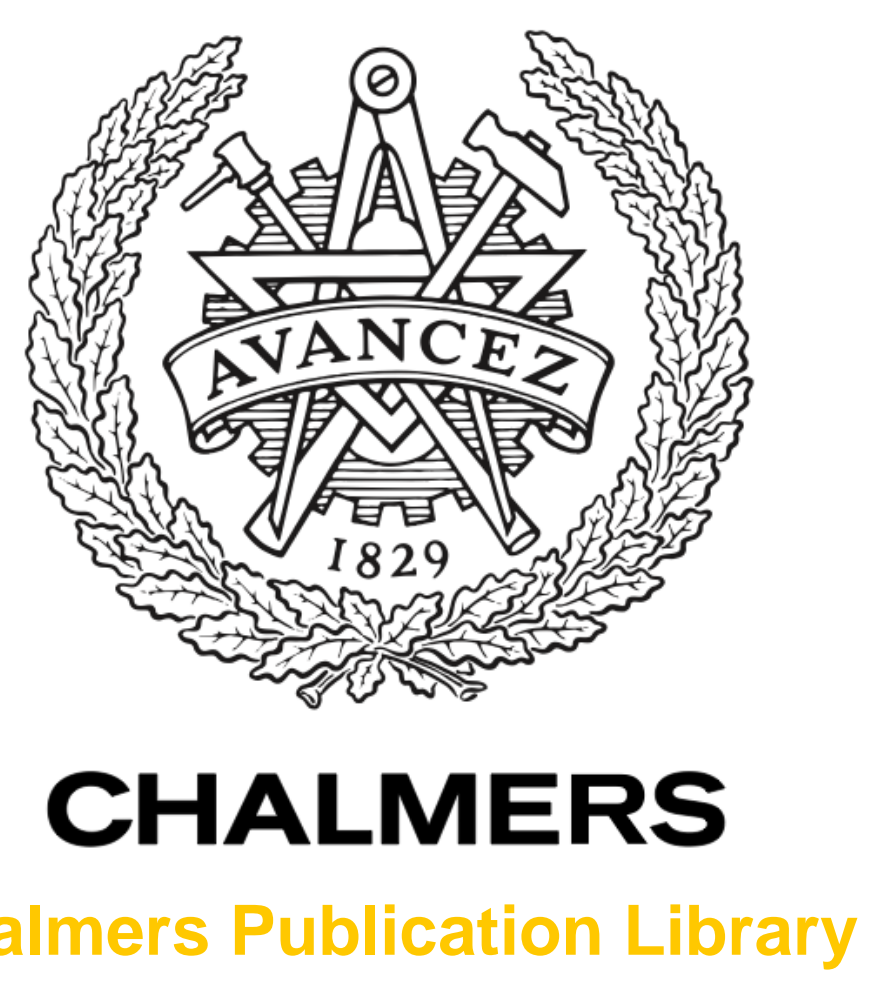

Chalmers Publication Library

\title{
Robust LPV-based infinite horizon LQR design
}

This document has been downloaded from Chalmers Publication Library (CPL). It is the author's version of a work that was accepted for publication in:

Proceedings of the 2017 21st International Conference on Process Control (PC)

Citation for the published paper:

Ilka, A. ; Veselý, V. (2017) "Robust LPV-based infinite horizon LQR design". Proceedings of the 2017 21st International Conference on Process Control (PC) pp. 86-91.

Downloaded from: http://publications.lib.chalmers.se/publication/249768

Notice: Changes introduced as a result of publishing processes such as copy-editing and formatting may not be reflected in this document. For a definitive version of this work, please refer to the published source. Please note that access to the published version might require a subscription. 


\section{Robust LPV-based infinite horizon LQR design*}

\author{
Adrian Ilka \\ Department of Signals and Systems \\ Chalmers Universiy of Technology \\ SE-412 96, Gothenburg, Sweden \\ Email: adrian.ilka@chalmers.se
}

\author{
Vojtech Veselý \\ Institute of Robotics and Cybernetics \\ Slovak University of Technology in Bratislava \\ Ilkovičcova 3, 81219 Bratislava \\ Email: vojtech.vesely@stuba.sk
}

\begin{abstract}
In this paper, the problem of robust infinite horizon linear quadratic regulator (LQR) design is addressed for uncertain affine linear parameter-varying (LPV) systems. The proposed method extends the standard infinite horizon LQR design to LPV-based static output-feedback (SOF), dynamic outputfeedback (DOF) and to a well known proportional, integral and derivative (PID) controller design for uncertain affine LPV systems. The optimal (suboptimal) controller design is formulated as an optimization problem subject to some linear/bilinear matrix inequality (LMI/BMI) constraints. As the main result, the suggested performance and stability conditions, without any restriction on the controller and system structure, are convex functions of the scheduling and uncertainty parameters. Hence, there is no need for applying multi-convexity or other relaxation techniques and consequently the proposed solution delivers a less conservative design method. The viability of the novel design technique is demonstrated and evaluated through numerical examples.
\end{abstract}

\section{INTRODUCTION}

One of the most fundamental problems in control theory is the linear quadratic regulator (LQR) design problem [1]. The so-called infinite horizon linear quadratic problem of finding a control function $u^{*} \in \mathbb{R}^{m}$ for $x_{0} \in \mathbb{R}^{n}$ that minimizes the cost functional:

$$
\begin{aligned}
J^{*}=\int_{0}^{\infty}\left(x(t)^{T} Q x(t)\right. & +u^{T}(t) R u(t) \\
& \left.+2 x^{T}(t) N u(t)\right) d t,
\end{aligned}
$$

with $R>0, Q-N R^{-1} N^{T} \geq 0$ subject to $\dot{x}(t)=A x(t)+$ $B u(t), x(0)=x_{0}$ has been studied by many authors [1], [2], [3], [4]. In many cases, it is not possible or economically feasible to measure all the state variables. This resulted in several generalized versions of the above problem to outputfeedback with both necessary and sufficient conditions for the existence of a solution [5], [6], [7]. The well known proportional, integral and derivative (PID) equivalent of a LQR can be also found in [8].

Subsequently, in many papers have been studied the robust static output-feedback version of the LQR design [9], [10], [11], as well as the LQR-based PID controller design [12], [13], [14]. The introduction of the linear parametervarying (LPV) systems [15] has opened new possibilities in LQR design. Several gain-scheduled/LPV-based LQR design

*This work was supported by the Chalmers Area of Advance Transportation, by Vinnova under the FFI projects MultiMEC and VCloud II, and by Scientific Grant Agency VEGA (1/0475/16) which is gratefully acknowledged. techniques appeared in both static output feedback (SOF) and dynamic output-feedback (DOF), not to mention the PID controller design [16], [17], [18], [19], [20], [21], [22].

Nonetheless, all of the mentioned approaches have some limitations. The majority of these approaches uses the multiconvexity lemma [23] to obtain a finite number of linear/bilinear matrix inequalities (LMI/BMI). This is introducing some conservativeness in the controller design, since it forces the Bellman-Lyapunov function to be multi-convex. Thus, different relaxation techniques have been deployed to reduce the conservativeness caused by the multi-convexity requirement [18], [19], [24]. Nevertheless, these relaxations can have significant influence on the performance and can drift the guaranteed cost far away from its optima. Multi-convexity has been differently solved, usually by restricting the closedloop LPV structure, system or controller to avoid cross term effects of the scheduling parameters [21], [22].

From this short literature survey follows that there is no general LPV-based LQR controller design approach, which gives convex dependency on scheduling parameters without major restrictions on the closed-loop system or controller matrices. Therefore, in this paper we present a generalized LPV-based LQR design for uncertain affine LPV systems which allows beside SOF and DOF the well known PID controller structure as well. Furthermore, the suggested performance and stability conditions, without any restriction on the controller and system structure, are convex functions of the scheduling and uncertain parameters. Hence, there is no need for applying the multiconvexity or other relaxation techniques.

The rest of the paper is organized into four sections. The introduction is followed by preliminaries and problem formulation in Section 2. The main result is presented in Section 3, where the generalized LPV-based LQR design is presented. Numerical examples are given in Section 4. Concluding remarks close the paper in Section 5.

The mathematical notation of the paper is as follows. Given a symmetric matrix $P=P^{T} \in \mathbb{R}^{n \times n}$, the inequality $P>0$ $(P \geq 0)$ denotes the positive definiteness (semi definiteness) of the matrix. Matrices, if not explicitly stated, are assumed to have compatible dimensions. I denotes the identity matrix of corresponding dimensions. The symbol $*$ denotes a block that is readily inferred by symmetry. Notation for interval of numbers between $a$ and $b$ including endpoints $a$ and $b$ is $\langle a, b\rangle=\{x \in \mathbb{R} \mid a \leq x \leq b\}$. 


\section{PRELIMINARIES AND PROBLEM FORMULATION}

Consider the following uncertain LPV system as follows:

$$
\begin{aligned}
E(\theta(t)) \dot{x}(t) & =A(\theta(t)) x(t)+B(\theta(t)) u(t), \\
y(t) & =C(\theta(t)) x(t)+D(\theta(t)) u(t) .
\end{aligned}
$$

where $x(t) \in \mathbb{R}^{n}, y(t) \in \mathbb{R}^{l}$, and $u(t) \in \mathbb{R}^{m}$ are the state, measurable output, and the control input, respectively. The matrix functions $E(\theta(t)) \in \mathbb{R}^{n \times n}, A(\theta(t)) \in \mathbb{R}^{n \times n}$, $B(\theta(t)) \in \mathbb{R}^{n \times m}, C(\theta(t)) \in \mathbb{R}^{l \times n}$ and $D(\theta(t)) \in \mathbb{R}^{l \times m}$ are assumed to depend on the scheduling variable $\theta(t) \in\langle\underline{\theta}, \bar{\theta}\rangle \in$ $\Omega$ as (3) with $S(\theta(t))=\{E(\theta(t)), A(\theta(t)), B(\theta(t)), C(\theta(t))$, $D(\theta(t))\}$.

$$
\begin{aligned}
S(\theta(k)) & =S_{0}+\sum_{i=1}^{N_{\alpha}} S_{i} \alpha_{i}(k)+\sum_{j=1}^{N_{\beta}} S_{\left(N_{\alpha}+j\right)} \beta_{j}(k) \\
& +\sum_{g=1}^{N_{\delta}} S_{\left(N_{\alpha}+N_{\beta}+g\right)} \delta_{g}(k)=S_{0}+\sum_{i=1}^{p} S_{i} \theta_{i}(k) .
\end{aligned}
$$

The scheduling variable used in this paper is extended and distributed to:

$$
\theta(k)=\left[\alpha_{1}, \ldots, \alpha_{N_{\alpha}}, \beta_{1}, \ldots, \beta_{N_{\beta}}, \delta_{1}, \ldots, \delta_{N_{\delta}}\right],
$$

where

a) it is assumed that the scheduling parameters $\alpha_{i}(k) i=$ $1,2, \ldots, N_{\alpha}$ are constant or time-varying, furthermore can be measured or estimated and therefore used in the controller,

b) the scheduling parameters $\beta_{j}(k), j=1,2, \ldots, N_{\beta}$ are constant or time-varying but unknown (uncertain) parameters,

c) $\delta_{g}(k), g=1,2, \ldots, N_{\delta}$ represents the uncertain part of the measurable/estimable scheduled parameters (see $A p$ pendix A),

It is assumed that the maximal rate of change of scheduled parameters $\max \dot{\theta}_{i}(t) \leq \rho_{\theta_{i}}$ are known and predefined. Furthermore, the matrix $E(\theta(t))$ is non-singular.

We are looking for a static output-feedback defined as

$$
u(t)=F(\theta(t)) y(t)
$$

where

$$
F(\theta(t))=F_{0}+\sum_{i=1}^{p} F_{i} \theta_{i}(t) \in \mathbb{R}^{m \times l} .
$$

Notice, that the controller gain matrices $F_{i}$ related to the uncertain parameters $\beta(t)$ and $\delta(t)$ are equal to zero.

The control law (5) can be augmented with integral part [14] for PI controller design. In this case the augmented system is defined as:

$$
\begin{aligned}
\bar{E}(\theta(t)) \dot{\bar{x}}(t) & =\bar{A}(\theta(t)) \bar{x}(t)+\bar{B}(\theta(t)) \bar{u}(t), \\
\bar{y}(t) & =\bar{C}(\theta(t)) \bar{x}(t)+\bar{D}(\theta(t)) \bar{u}(t),
\end{aligned}
$$

where $\bar{x}(t)^{T}=[x(t), z(t)] \in \mathbb{R}^{n+l}$ is the augmented state vector, $\bar{y}(t)^{T}=[y(t), z(t)] \in \mathbb{R}^{l+l}$ is the augmented output vector, and $\bar{u}(t)=u(t) \in \mathbb{R}^{m}$ is the augmented input vector. Furthermore, the augmented system and control matrices are:

$$
\begin{gathered}
\bar{E}(\theta(t))=\left[\begin{array}{cc}
E(\theta(t)), 0 \\
0, & I
\end{array}\right], \bar{A}(\theta(t))=\left[\begin{array}{c}
A(\theta(t)), 0 \\
C(\theta(t)), 0
\end{array}\right], \\
\bar{B}(\theta(t))=\left[\begin{array}{c}
B(\theta(t)) \\
0
\end{array}\right], \bar{C}(\theta(t))=\left[\begin{array}{cc}
C(\theta(t)), 0 \\
0,
\end{array}\right], \\
\bar{D}(\theta(t))=\left[\begin{array}{c}
D(\theta(t)) \\
0
\end{array}\right], \bar{F}(\theta(t))=\left[F(\theta(t)), F_{i}(\theta(t))\right] .
\end{gathered}
$$

We can design a full/reduced order dynamic output-feedback controller with order $n_{k}$, defined as:

$$
\begin{gathered}
\dot{x}_{c}=A_{c}(\theta(t)) x_{c}(t)+B_{c}(\theta(t)) y(t), \\
u(t)=C_{c}(\theta(t)) x_{c}(t)+D_{c}(\theta(t)) y(t), \\
x_{c}(t) \in \mathbb{R}^{n_{k}}, A_{c}(\theta(t)) \in \mathbb{R}^{n_{k} \times n_{k}}, B_{c}(\theta(t)) \in \mathbb{R}^{n_{k} \times l}, \\
C_{c}(\theta(t)) \in \mathbb{R}^{m \times n_{k}}, D_{c}(\theta(t)) \in \mathbb{R}^{m \times l},
\end{gathered}
$$

since the system (2) can be reformulated to static outputfeedback design, too [25]. In this case, the controller gain matrices affinely depend on the scheduling variable as (3) with $S(\theta(t))=\left\{A_{c}(\theta(t)), B_{c}(\theta(t)), C_{c}(\theta(t)), D_{c}(\theta(t))\right\}$ and the augmented system matrices are as follows:

$$
\begin{aligned}
& \bar{E}(\theta(t))=\left[\begin{array}{cc}
E(\theta(t)) & 0 \\
0, & I
\end{array}\right], \bar{A}(\theta(t))=\left[\begin{array}{cc}
A(\theta(t)) & 0 \\
0, & 0
\end{array}\right], \\
& \bar{B}(\theta(t))=\left[\begin{array}{cc}
B(\theta(t)) & 0 \\
0, & I
\end{array}\right], \bar{C}(\theta(t))=\left[\begin{array}{cc}
C(\theta(t)) & 0 \\
0, & I
\end{array}\right], \\
& \bar{D}(\theta(t))=\left[\begin{array}{cr}
D(\theta(t)) & 0 \\
0, & 0
\end{array}\right] \text {. }
\end{aligned}
$$

Furthermore, $\bar{x}(t)^{T}=\left[x(t), x_{c}(t)\right] \in \mathbb{R}^{n+n_{k}}$ is the augmented state vector, $\bar{y}(t)^{T}=\left[y(t), x_{c}(t)\right] \in \mathbb{R}^{l+n_{k}}$ is the augmented output vector, and $\bar{u}(t)^{T}=\left[u(t), x_{c}(t)\right] \in \mathbb{R}^{m+n_{k}}$ is the augmented input vector. The augmented feedback gain is then:

$$
\bar{F}(\theta(t))=\left[\begin{array}{l}
D_{c}(\theta(t)), C_{c}(\theta(t)) \\
B_{c}(\theta(t)), A_{c}(\theta(t))
\end{array}\right] .
$$

To obtain a PID controller design in the form:

1) PID controller with filter on the derivative part with filter time constant $T_{f d}$ :

$$
\begin{aligned}
u(t)=K_{p}(\theta(t)) y(t) & +K_{i}(\theta(t)) \int_{0}^{\infty} y(t) d t \\
& +K_{d}(\theta(t)) \frac{1}{T_{f d} s+1} \dot{y}(t),
\end{aligned}
$$

2) PID controller with filtered input with filter time constant $T_{f}$ :

$$
\begin{aligned}
u(t)= & \frac{1}{T_{f} s+1}\left(K_{p}(\theta(t)) y(t)\right. \\
& \left.+K_{i}(\theta(t)) \int_{0}^{\infty} y(t) d t+K_{d}(\theta(t)) \dot{y}(t)\right),
\end{aligned}
$$


one can prescribe the structure of the dynamic output-feedback controller matrices form (9) as follows:

$$
\begin{gathered}
A_{c}=\left[\begin{array}{ccc}
A_{c_{11}} & \ldots & A_{c_{1 m}} \\
\vdots & \ddots & \vdots \\
A_{c_{m 1}} & \ldots & A_{c_{m m}}
\end{array}\right], B_{c}=\left[\begin{array}{ccc}
B_{c_{11}} & \ldots & B_{c_{1 l}} \\
\vdots & \ddots & \vdots \\
B_{c_{m 1}} & \ldots & B_{c_{m l}}
\end{array}\right], \\
C_{c}=\left[\begin{array}{ccc}
C_{c_{11}} & \ldots & C_{c_{1 m}} \\
\vdots & \ddots & \vdots \\
C_{c_{m 1}} & \ldots & C_{c_{m m}}
\end{array}\right], D_{c}=\left[\begin{array}{ccc}
D_{c_{11}} & \ldots & D_{c_{1 l}} \\
\vdots & \ddots & \vdots \\
D_{c_{m 1}} & \ldots & D_{c_{m l}}
\end{array}\right],
\end{gathered}
$$

where the matrices $A_{c_{i j}} \in \mathbb{R}^{2 \times 2}, B_{c_{i j}} \in \mathbb{R}^{2 \times 1}, C_{c_{i j}} \in \mathbb{R}^{1 \times 2}$, and $D_{c_{i j}} \in \mathbb{R}^{1 \times 1}$ are:

$$
\begin{gathered}
A_{c_{i j}}=\left[\begin{array}{l}
-a_{1}, 1 \\
-a_{2}, 0
\end{array}\right], B_{c_{i j}}=\left[\begin{array}{c}
b_{1_{i j}}(\theta(t))-a_{1} b_{0_{i j}}(\theta(t)) \\
b_{2_{i j}}\left(\theta(t)-a_{2} b_{0_{i j}}(\theta(t))\right.
\end{array}\right] \\
C_{c_{i j}}=[1,0], D_{c_{i j}}=\left[b_{0_{i j}}(\theta(t))\right]
\end{gathered}
$$

where

1) For the PID controller with filter on the derivative part:

$$
\begin{gathered}
b_{0_{i j}}(\theta(t))=\frac{k_{p_{i j}}(\theta(t)) T_{f d}+k_{d_{i j}}(\theta(t))}{T_{f d}}, \\
b_{1_{i j}}(\theta(t))=\frac{k_{p_{i j}}(\theta(t))+k_{i_{i j}}(\theta(t)) T_{f d}}{T_{f d}}, \\
b_{2_{i j}}(\theta(t))=\frac{k_{i_{i j}}(\theta(t))}{T_{f d}}, a_{1}=\frac{1}{T_{f d}}, a_{2}=0 .
\end{gathered}
$$

2) For the PID controller with filtered input:

$$
\begin{gathered}
b_{0_{i j}}(\theta(t))=\frac{k_{d_{i j}}(\theta(t))}{T_{f}}, b_{1_{i j}}(\theta(t))=\frac{k_{p_{i j}}(\theta(t))}{T_{f}}, \\
b_{2_{i j}}(\theta(t))=\frac{k_{i_{i j}}(\theta(t))}{T_{f}}, a_{1}=\frac{1}{T_{f}}, a_{2}=0 .
\end{gathered}
$$

The controller gain matrices are affinely dependent on the scheduling variable as (3) with $S(\theta(t))=\left\{K_{p}(\theta(t))\right.$, $\left.K_{i}(\theta(t)), K_{d}(\theta(t))\right\} \in \mathbb{R}^{m \times l}$. For centralized controller design the gain matrices $K_{p}(\theta(t)), K_{i}(\theta(t))$ and $K_{d}(\theta(t))$ are full matrices:

$$
K_{x}(\theta(t))=\left[\begin{array}{ccc}
k_{x_{11}}(\theta(t)) & \ldots & k_{x_{1 l}}(\theta(t)) \\
\vdots & \ddots & \vdots \\
k_{x_{m 1}}(\theta(t)) & \ldots & k_{x_{m l}}(\theta(t))
\end{array}\right], x=p, i, d .
$$

To obtain decentralized control, the structure of these matrices can be predefined. In the case when $m=l$ a fully decentralized control can be obtained by structuring the gain matrices to diagonal form.

The main goal of this paper is to design a controller (in the form (5)) which guarantees the closed-loop stability and minimizes the cost function defined as:

$$
J_{\infty}=\int_{0}^{\infty} J(\theta(t)) d t=\int_{0}^{\infty} \tilde{z}^{T} Q(\theta(t)) \tilde{z} d t
$$

where $\tilde{z}^{T}=[\dot{x}(t), x(t), u(t), y(t)] \in \mathbb{R}^{2 n+m+l}$ and

$$
Q(\theta(t))=\left[\begin{array}{cccc}
Q_{\dot{x}}(\theta), & N_{\dot{x} x}(\theta), & N_{\dot{x} u}(\theta), & N_{\dot{x} y}(\theta) \\
*, & Q_{x}(\theta), & N_{x u}(\theta), & N_{x y}(\theta) \\
*, & *, & R(\theta), & N_{u y}(\theta) \\
*, & *, & *, & Q_{y}(\theta)
\end{array}\right] \geq 0 .
$$

The weighting matrix $Q(\theta(t))$ is affinely dependent on the scheduling variable defined as (3) with $S(\theta(t))=\{Q(\theta(t))\}$. In addition, $Q_{\dot{x}}(\theta(t)) \in \mathbb{R}^{n \times n} \geq 0, Q_{x}(\theta(t)) \in \mathbb{R}^{n \times n}$, $Q_{y}(\theta(t)) \in \mathbb{R}^{l \times l}, R(\theta(t)) \in \mathbb{R}^{m \times m}>0$ and matrices $N_{i j}(\theta(t)), i, j=\dot{x}, x, u, y, i \neq j$ are cross-term weighting matrices with appropriate dimensions.

Remark 1. Controllability and observability analysis for LPV systems can be done by using the approach presented in [26].

\section{ROBUST LPV BASED LQR DESIGN}

The main result of this paper is formulated in the next theorem. For the shake of simplicity, all the undefined expressions will be explained in the proof of the next theorem.

Theorem 1. For the uncertain affine LPV system (2) an optimal (suboptimal) LQR-based controller exists in the form (5), if for the given weighting matrix $Q(\theta(t))$ and predefined maximal rate of change of scheduled parameters $\rho_{\theta}$, the following conditions hold:

$$
\min _{F, P, X} \operatorname{trace}\left(\sum_{i=1}^{2^{p}} \tilde{P}_{i}\right)
$$

subject to:

$$
\begin{gathered}
\tilde{M}_{i}=\left[\begin{array}{ccc}
\tilde{M}_{i_{11}} & \ldots & \tilde{M}_{i_{41}}^{T} \\
\vdots & \ddots & \vdots \\
\tilde{M}_{i_{41}} & \ldots & \tilde{M}_{i_{44}}
\end{array}\right] \leq 0 \\
\tilde{P}_{i}>0 \\
i=1,2, \ldots, 2^{p}
\end{gathered}
$$

where

$$
\begin{aligned}
\tilde{M}_{i_{11}}= & \tilde{E}_{i}^{T} X_{1}+X_{1}^{T} \tilde{E}_{i}+\tilde{S}_{i}, \\
\tilde{M}_{i_{21}}= & -\tilde{A}_{i}^{T} X_{1}+X_{2}^{T} \tilde{E}_{i}-\tilde{C}^{T} X_{9}+\tilde{N}_{i_{\dot{x} x}}^{T}+\tilde{P}_{i}, \\
\tilde{M}_{i_{31}}= & -\tilde{B}_{i}^{T} X_{1}+X_{3}^{T} \tilde{E}_{i}+X_{5}-\tilde{D}_{i}^{T} X_{9}+\tilde{N}_{i_{\dot{x} u}}^{T}, \\
\tilde{M}_{i_{41}}= & X_{4}^{T} \tilde{E}_{i}-\tilde{F}_{i}^{T} X_{5}+X_{9}+\tilde{N}_{i_{\dot{x} y}}^{T}, \\
\tilde{M}_{i_{22}}= & -X_{2}^{T} \tilde{A}_{i}-\tilde{A}_{i}^{T} X_{2}-X_{10} \tilde{C}_{i}-\tilde{C}_{i}^{T} X_{10}+\tilde{Q}_{i_{x}}+P_{\rho}, \\
\tilde{M}_{i_{32}}= & -\tilde{B}_{i}^{T} X_{2}-X_{3}^{T} \tilde{A}_{i}+X_{6}-\tilde{D}_{i}^{T} X_{10} \\
& -X_{11}^{T} \tilde{C}_{i}+\tilde{N}_{i_{x u}}^{T}, \\
\tilde{M}_{i_{42}}= & -X_{4}^{T} \tilde{A}_{i}-\tilde{F}_{i}^{T} X_{6}+X_{10}-X_{12}^{T} \tilde{C}_{i}+\tilde{N}_{i_{x y}}^{T}, \\
\tilde{M}_{i_{33}}= & -X_{3}^{T} \tilde{B}_{i}-\tilde{B}_{i}^{T} X_{3}+X_{7}^{T}+X_{7}-X_{11}^{T} \tilde{D}_{i} \\
& -\tilde{D}_{i}^{T} X_{11}+\tilde{R}_{i}, \\
\tilde{M}_{i_{43}}= & -X_{4}^{T} \tilde{B}_{i}-\tilde{F}_{i}^{T} X_{7}+X_{8}^{T}+X_{11} \\
& -X_{12}^{T} \tilde{D}_{i}+\tilde{N}_{i_{u y}}^{T}, \\
\tilde{M}_{i_{44}}= & -X_{8}^{T} \tilde{F}_{i}-\tilde{F}_{i}^{T} X_{8}+X_{12}^{T}+X_{12}+\tilde{Q}_{i_{y}} .
\end{aligned}
$$

Proof. The proof is based on the free matrix weighting approach:

$$
\begin{gathered}
2\left(\dot{x}^{T} X_{1}^{T}+x^{T} X_{2}^{T}+u^{T} X_{3}^{T}+y^{T} X_{4}^{T}\right) \\
(\dot{x}-A(\theta) x-B(\theta) u)=0, \\
2\left(\dot{x}^{T} X_{5}^{T}+x^{T} X_{6}^{T}+u^{T} X_{7}^{T}+y^{T} X_{8}^{T}\right) \\
(y-C(\theta) x-D(\theta) u)=0, \\
2\left(\dot{x}^{T} X_{9}^{T}+x^{T} X_{10}^{T}+u^{T} X_{11}^{T}+y^{T} X_{12}^{T}\right) \\
(u-F(\theta) y)=0 .
\end{gathered}
$$


The matrices $X_{i}, i=1, \ldots, 12$ are auxiliary matrices with appropriate dimensions. One can transform the equalities (21)(23) to the form:

$$
\tilde{z}^{T}(t) W(\theta) \tilde{z}(t)=0
$$

where

$$
\begin{aligned}
W_{11}= & E(\theta)^{T} X_{1}+X_{1}^{T} E(\theta), \\
W_{21}= & -A(\theta)^{T} X_{1}+X_{2}^{T} E(\theta)-C(\theta)^{T} X_{9}, \\
W_{31}= & -B(\theta)^{T} X_{1}+X_{3}^{T} E(\theta)+X_{5}-D(\theta)^{T} X_{9}, \\
W_{41}= & X_{4}^{T} E(\theta)-F(\theta)^{T} X_{5}+X_{9}, \\
W_{22}= & -X_{2}^{T} A(\theta)-A(\theta)^{T} X_{2}-X_{10}^{T} C(\theta) \\
& -C(\theta)^{T} X_{10}, \\
W_{32}= & -B(\theta)^{T} X_{2}-X_{3}^{T} A(\theta)+X_{6}-D(\theta)^{T} X_{10} \\
& -X_{11}^{T} C(\theta), \\
W_{42}= & -X_{4}^{T} A(\theta)-F(\theta)^{T} X_{6}+X_{10}-X_{12}^{T} C(\theta), \\
W_{33}= & -X_{3}^{T} B(\theta)-B(\theta)^{T} X_{3}+X_{7}^{T}+X_{7} \\
& -X_{11}^{T} D(\theta)-D(\theta)^{T} X_{11}, \\
W_{43}= & -X_{4}^{T} B(\theta)-F(\theta)^{T} X_{7}+X_{8}^{T}+X_{11} \\
& -X_{12}^{T} D(\theta), \\
W_{44}= & -X_{8}^{T} F(\theta)-F(\theta)^{T} X_{8}+X_{12}^{T}+X_{12} .
\end{aligned}
$$

Let's choose the Lyapunov function as:

$$
V(\theta(t))=x(t)^{T} P(\theta(t)) x(t)
$$

where

$$
P(\theta(t))=P_{0}+\sum_{i=1}^{p} P_{i} \theta_{i}(t) .
$$

The first derivative of the Lyapunov function (25) is then:

$$
\begin{aligned}
\dot{V}(\theta(t))=\dot{x}^{T}(t) P(\theta(t)) x(t) & +x^{T}(t) P(\theta(t)) \dot{x}(t) \\
& +x^{T}(t) P(\dot{\theta}(t)) x(t) .
\end{aligned}
$$

where

$$
P(\dot{\theta}(t)) \leq \sum_{i=1}^{p} P_{i} \rho_{i}=P_{\rho} .
$$

One can transform the first derivative of the Lyapunov function (27) with (28) to the form:

$$
\dot{V}(\theta(t))=\tilde{z}^{T}(t)\left[\begin{array}{ccc}
0, & P(\theta(t)), & 0,0 \\
P(\theta(t)), & P_{\rho}, & 0,0 \\
0, & 0, & 0,0 \\
0, & 0, & 0,0
\end{array}\right] \tilde{z}(t) .
$$

By summarizing the equations (17), (24) and (29), the Bellman-Lyapunov inequality can be obtained in the form:

$$
\begin{aligned}
\tilde{z}^{T}(t)(W(\theta(t))+\dot{V}(\theta(t)) & +J(\theta(t))) \tilde{z}(t) \\
& =\tilde{z}^{T}(t) M(\theta(t)) \tilde{z}(t) \leq 0,
\end{aligned}
$$

Furthermore, if $P(\theta(t))$ is positive definite then the BellmanLyapunov inequality (30) can be rewritten to this form $(W(\theta(t))=0)$ :

$$
\dot{V}(\theta(t))+J(\theta(t)) \leq 0 \rightarrow \dot{V}(\theta(t)) \leq-J(\theta(t)) \leq 0
$$

Integrating both side form 0 to $\infty$ one can obtain:

$$
J_{\infty}=\int_{0}^{\infty} J(\theta(t)) \leq V(0)-V(\infty)=x_{0}^{T} P(\theta(0)) x_{0} .
$$

It follows that by minimizing $x_{0}^{T} P(\theta(0)) x_{0}$ and by satisfying $M(\theta(t)) \leq 0$ as well as $P(\theta(t))>0$ for all $\theta \in \Omega$, the closed-loop system will be affinely quadratically stable with guaranteed cost defined by (32).

Since $M(\theta(t))$ and $P(\theta(t))$ are convex regarding the scheduling variable $\theta$, therefore $M(\theta(t))$ will be negative semi-definite and $P(\theta(t))$ will be positive definite if and only if they are negative semi-definite and definite at the corners of $\theta$. Hence, definiteness/semi-definiteness splits to $2 \times 2^{p}$ inequalities $\rightarrow$ (19) and (20). The tilde denotes the given elements at the vertices of $\theta$.

If the initial condition of the scheduling variable is available then it is enough to minimize $\operatorname{trace}(P(\theta(0)))$. Otherwise we recommend to minimize: $\operatorname{trace}\left(\sum_{i=1}^{2^{p}} \tilde{P}_{i}\right)$.

\section{EXAMPLES}

In order to show the viability of the previous proposed method, the following two examples have been chosen.

Example 1. The first example is a simple multi-input multioutput (MIMO) linear time-invariant (LTI) system, which will be used to demonstrate and compare the proposed method with the standard LQR design. The transfer function of the system is as follows:

$$
G(s)=\left[\begin{array}{cc}
\frac{2}{10 s+1}, & \frac{2}{s+1} \\
\frac{1}{s+1}, & \frac{-4}{10 s+1}
\end{array}\right],
$$

which can be transformed to the form (2):

$$
\begin{aligned}
& A=\left[\begin{array}{cccc}
-0.1, & 0, & 0, & 0 \\
0, & -1, & 0, & 0 \\
0, & 0, & -1, & 0 \\
0, & 0, & 0, & -0.1
\end{array}\right], B=\left[\begin{array}{cc}
0.5, & 0 \\
1, & 0 \\
0, & 2 \\
0, & 0.5
\end{array}\right] \\
& C=\left[\begin{array}{c}
0.4,0,1,0 \\
0,1,0,-0.8
\end{array}\right], D=\left[\begin{array}{l}
0,0 \\
0,0
\end{array}\right], E=\left[\begin{array}{l}
1,0,0,0 \\
0,1,0,0 \\
0,0,1,0 \\
0,0,0,1
\end{array}\right]
\end{aligned}
$$

Different controller types were designed: static state feedback (SSF), centralized/decentralized SOF, reduced/full order dynamic state feedback (DSF), reduced/full order DOF, centralized/decentralized PI and centralized/decentralized PID. Numerical solution has been carried out by PENBMI 2.1 [27] / PENLAB 1.04 [28] solver under MATLAB 2014b using YALMIP R20150918 [29]. The simulations were done via SIMULINK. The obtained guaranteed cost $\left(J_{\infty}\right)$ for:

$$
\begin{gathered}
Q_{x}=\left[\begin{array}{l}
1,0,0,0 \\
0,1,0,0 \\
0,0,1,0 \\
0,0,0,1
\end{array}\right], R=\left[\begin{array}{l}
1,0 \\
0,1
\end{array}\right], N_{x u}=\left[\begin{array}{l}
0.1,0.1 \\
0.1,0.1 \\
0.1,0.1 \\
0.1,0.1
\end{array}\right], \\
Q_{y}, Q_{\dot{x}}, N_{i, j}=0, i, j=\dot{x}, x, y, u ; i \neq j ; i, j \neq x, u
\end{gathered}
$$

can be found in Table 1 . Similar comparison with output weighting can be found in Table 2 for:

$$
\begin{gathered}
Q_{y}=\left[\begin{array}{l}
1,0 \\
0,1
\end{array}\right], R=\left[\begin{array}{l}
1,0 \\
0,1
\end{array}\right], N_{u y}=\left[\begin{array}{l}
0.1,0.1 \\
0.1,0.1
\end{array}\right], \\
Q_{x}, Q_{\dot{x}}, N_{i, j}=0, i, j=\dot{x}, x, y, u ; i \neq j ; i, j \neq u, y
\end{gathered}
$$

Example 2. The second example is an arm-driven inverted pendulum (ADIP) with strong nonlinearity described in [30] 
TABLE I

CONTROLLER TYPES \& GUARANTEED COSTS

\begin{tabular}{|c|c|}
\hline Controller type & $J_{\infty}$ \\
\hline \multicolumn{2}{|l|}{ Standard infinite-horizon $L Q R$ : } \\
\hline$\triangle \mathrm{SSF}$ & 3.6913 \\
\hline$\triangleright \mathrm{DSF}$ & 3.6749 \\
\hline \multicolumn{2}{|l|}{ Proposed method: } \\
\hline$\triangleright$ SSF & 3.6913 \\
\hline$\triangleright$ Centralized SOF & 4.9736 \\
\hline$\triangleright$ Decentralized SOF & 5.8906 \\
\hline$\triangleright \operatorname{DSF}\left(n_{k}=4\right)$ & 3.6749 \\
\hline$\triangleright \operatorname{DOF}\left(n_{k}=2\right)$ & 4.9699 \\
\hline$\triangleright$ Centralized PI & 9.9376 \\
\hline$\triangleright$ Decentralized PI & 13.2005 \\
\hline$\triangleright$ Decentralized PID $_{1}\left(T_{f d}=0.5\right)$ & 11.3854 \\
\hline$\triangleright$ Decentralized $\mathrm{PID}_{2}\left(T_{f}=0.5\right)$ & 11.3854 \\
\hline
\end{tabular}

CONTROLlER TYPES \& GUARANTEED COSTS

\begin{tabular}{lc} 
Controller type & $J_{\infty}$ \\
\hline Standard infinite-horizon LQR with output weighting: & \\
$\quad \triangleright$ SSF & 1.0013 \\
$\quad \triangleright$ DSF & 0.9909 \\
Proposed method: & \\
$\quad \triangleright$ SSF & 1.0013 \\
$\quad \triangleright$ Centralized SOF & 1.1002 \\
$\quad \triangleright$ Decentralized SOF & 2.4490 \\
$\quad \triangleright$ DSF $\left(n_{k}=2\right)$ & 0.9962 \\
$\quad \triangleright$ DOF $\left(n_{k}=2\right)$ & 1.0026
\end{tabular}

(Fig. 1). The ADIP has two links with joints and encoders to measure the relative angles. The first joint is equipped with a geared DC motor. The main goal is to keep the second link in upright position for a given reference angle of the first joint. Furthermore, to ensure $|u| \leq 0.35[p . m]$ while the max step size for $\varphi_{1}$ can be $60[\mathrm{deg}]$.

The nonlinear model of the ADIP can be described with the following motion equation:

$$
\begin{gathered}
{\left[\begin{array}{cc}
\alpha_{1}, & \alpha_{3} \cos \varphi_{12} \\
\alpha_{3} \cos \varphi_{12}, & \alpha_{2}
\end{array}\right]\left[\begin{array}{l}
\ddot{\varphi}_{1} \\
\ddot{\varphi}_{2}
\end{array}\right]+\left[\begin{array}{c}
\alpha_{3} \dot{\varphi}_{2}^{2} \sin \varphi_{12} \\
-\alpha_{3} \dot{\varphi}_{1}^{2} \sin \varphi_{12}
\end{array}\right]} \\
+\left[\begin{array}{c}
-\alpha_{4} \sin \varphi_{1} \\
-\alpha_{5} \sin \varphi_{2}
\end{array}\right]+\left[\begin{array}{cc}
c_{1}+c_{2}, & -c_{2} \\
-c_{2}, & c_{2}
\end{array}\right]\left[\begin{array}{l}
\dot{\varphi}_{1} \\
\dot{\varphi}_{2}
\end{array}\right]=\left[\begin{array}{c}
\tau \\
0
\end{array}\right]
\end{gathered}
$$

where $\varphi_{12}=\varphi_{1}-\varphi_{2}, \alpha_{1}=J_{g 1}+m_{1} l_{1}^{2}+m_{2} L_{1}^{2}, \alpha_{2}=J_{g 2}+$ $m_{2} l_{2}^{2}, \alpha_{3}=m_{2} L_{1} l_{2}, \alpha_{4}=m_{1} g l_{1}+m_{2} g L_{1}, \alpha_{5}=m_{2} g l_{2}$, furthermore, $m_{i}$ is the $i$-th link's mass, $c_{i}$ is the viscous friction coefficient of the joint $i, J_{g i}$ is the moment of inertia about the center of gravity of the link $i, l_{i}$ is the length between the center of gravity of the link $i$ and the joint $i, L_{1}$ is the length between the fist and the second joint, and $g$ is the gravity acceleration. The physical parameters are shown in Table 3.

Since the active joint is operated by a geared DC motor and a velocity control driver, the model (36) can be rewritten to:

$$
\begin{aligned}
& {\left[\begin{array}{cc}
1, & 0 \\
\alpha_{3} \cos \varphi_{12}, \alpha_{2}
\end{array}\right]\left[\begin{array}{c}
\ddot{\varphi}_{1} \\
\ddot{\varphi}_{2}
\end{array}\right]+\left[\begin{array}{c}
0 \\
-\alpha_{3} \dot{\varphi}_{1}^{2} \sin \varphi_{12}
\end{array}\right]} \\
& +\left[\begin{array}{c}
0 \\
-\alpha_{5} \sin \varphi_{2}
\end{array}\right]+\left[\begin{array}{c}
a_{s}, \\
-c_{2}, c_{2}
\end{array}\right]\left[\begin{array}{c}
\dot{\varphi}_{1} \\
\dot{\varphi}_{2}
\end{array}\right]=\left[\begin{array}{c}
b_{s} \\
0
\end{array}\right] u
\end{aligned}
$$

where $u$ is the control input and $a_{s}=6.25, b_{s}=15.6$.

TABLE III

SYSTEM PARAMETERS

$$
\begin{array}{cl|cl}
m_{1}: & 3.06 \times 10^{-1}[\mathrm{~kg}] & m_{2}: & 1.04 \times 10^{-1}[\mathrm{~kg}] \\
l_{1}: & 1.20 \times 10^{-1}[\mathrm{~m}] & l_{2}: & 1.75 \times 10^{-1}[\mathrm{~m}] \\
L_{1}: & 2.27 \times 10^{-1}[\mathrm{~m}] & c_{2}: & 1.86 \times 10^{-4}\left[\mathrm{~kg} \cdot \mathrm{m}^{2} / \mathrm{s}\right] \\
J_{g 1}: & 1.38 \times 10^{-3}\left[\mathrm{~kg} \cdot \mathrm{m}^{2}\right] & J_{g 2}: & 1.38 \times 10^{-4}\left[\mathrm{~kg} \cdot \mathrm{m}^{2}\right]
\end{array}
$$

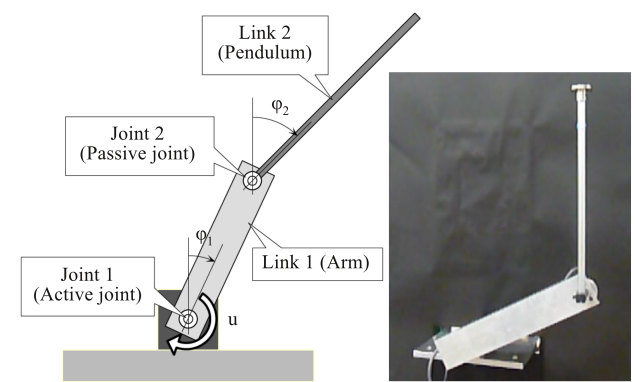

Fig. 1. Arm-driven inverted pendulum [30].

The model (37) can be rewritten to the form (2), with the assumption that $\sin \varphi_{2}=\varphi_{2}$ (since we assume that $\varphi_{2} \leq 2[\operatorname{deg}]$ ), with $x=\left[\varphi_{1}, \varphi_{2}, \omega_{1}, \omega_{2}\right]^{T}$ and with scheduled parameters (with assumption that $\varphi_{1} \leq 65[\mathrm{deg}]$ ) defined as $\theta_{1}=1-\cos \left(x_{1}-x_{2}\right) \in\langle 0,0.557\rangle, \theta_{2}=-\alpha_{3} x_{3} \sin \left(x_{1}-\right.$ $\left.x_{2}\right) \in\langle-0.003,0.003\rangle, \dot{\theta}_{1} \leq 0.4$ and $\dot{\theta}_{2} \leq 6 \times 10^{-} 3$.

$$
\begin{gathered}
A(\theta)=\left[\begin{array}{cccc}
0, & 0, & 1, & 0 \\
0, & 0, & 0, & 1 \\
0, & 0, & -a_{s}, & 0 \\
0, \alpha_{5}, & p_{2}(\theta),-c_{1}
\end{array}\right], B=\left[\begin{array}{c}
0 \\
0 \\
b_{s} \\
0
\end{array}\right], \\
C=\left[\begin{array}{l}
1,0,0,0 \\
0,1,0,0 \\
0,0,1,0 \\
0,0,0,1
\end{array}\right], D=\left[\begin{array}{l}
0,0 \\
0,0 \\
0,0 \\
0,0
\end{array}\right], E(\theta)=\left[\begin{array}{ccc}
1,0, & 0, & 0 \\
0,1, & 0, & 0 \\
0,0, & 1, & 0 \\
0,0, p_{1}(\theta), \alpha_{2}
\end{array}\right], \\
p_{1}(\theta)=\alpha_{3}-\alpha_{3} \theta_{1}, p_{2}(\theta)=c_{2}+\theta_{2} .
\end{gathered}
$$

The obtained model was extended for PI controller design as (7) with integral part for $\varphi_{1}$. Then a gain-scheduled PI controller was designed in the form (5) with weighting matrices:

$$
\begin{gathered}
Q_{x}(\theta)=\operatorname{diag}\left(\left[q_{1}(\theta), 1,0.1,0.1, q_{5}(\theta)\right]\right), R=1, \\
q_{1}(\theta)=2.8+2 \theta_{1}, q_{5}(\theta)=18-10 \theta_{2}, \\
Q_{y}, Q_{\dot{x}}, N_{i, j}=0, i, j=\dot{x}, x, y, u ; i \neq j .
\end{gathered}
$$

The obtained controller gain matrices are as follows:

$$
\begin{aligned}
& F_{0}=[12.1179,106.1869,7.0281,14.4041,8.8046], \\
& F_{1}=[-13.0849,-49.2797,-5.9001,-6.6199,-9.8506], \\
& F_{2}=1 \times 10^{3}[0.3627,2.8745,0.3010,0.3908,0.2484] .
\end{aligned}
$$

Numerical solution has been carried out by MOSEK LMI solver under MATLAB 2014b using YALMIP R20150918 [29] by reducing the BMI problem into iterative LMI (by fixing parameters related to controller matrices). The simulations (Fig. 2) were done via SIMULINK using the nonlinear model (36).

\section{CONCLUSION}

A novel approach for optimal/suboptimal LPV-based robust LQR design is presented in this paper for uncertain affine LPV systems with inexact scheduled parameters. The proposed design method extends the standard infinite-horizon LQR design to linear parameter-varying SOF, DOF, PI and PID controller design. Moreover, the obtained stability and performance requirements are convex functions of the scheduled parameters without any restriction on the controller and system 

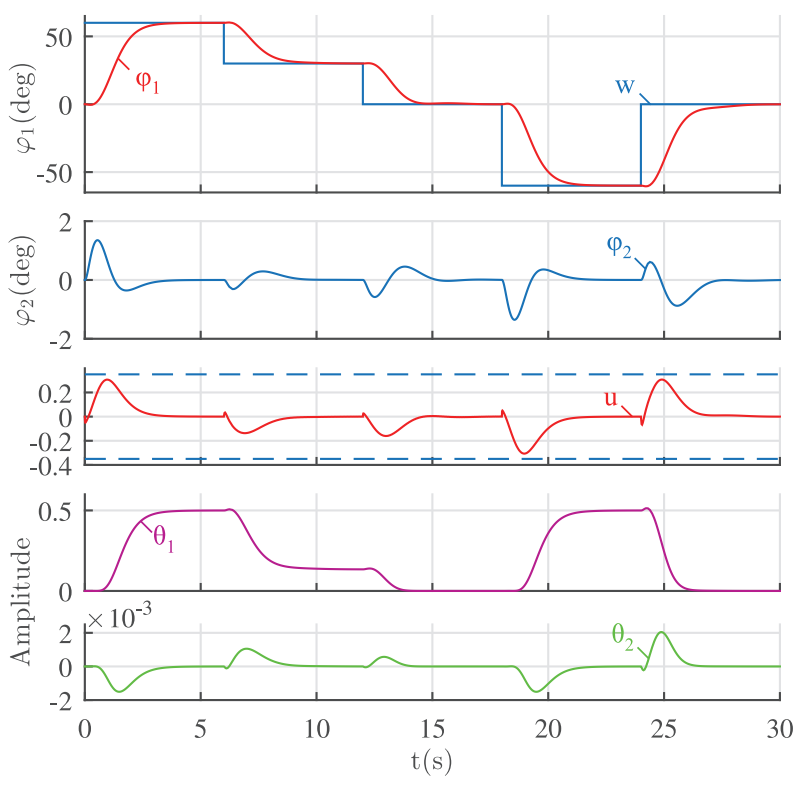

Fig. 2. Simulation results using the nonlinear model (36).

matrices. Therefore, the proposed approach delivers a less conservative and more usable design approach.

\section{APPENDIX A}

\section{Inexact scheduled parameters}

Assume that the measurable/estimable scheduling variable $\bar{\alpha}_{i}(t)$ consists of an exact part $\alpha_{i}(t)$ and from an error (inexact) part $\delta_{i}(t)$ :

$$
\bar{\alpha}_{i}(t)=\alpha_{i}(t)+\delta_{i}(t), i=1,2, \ldots, N_{\alpha} .
$$

For the affine form we get:

$$
\begin{aligned}
S(\bar{\alpha}(t)) & =S_{0}+\sum_{i=1}^{N_{\alpha}} S_{i}\left(\alpha_{i}(t)+\delta_{i}(t)\right) \\
& =S_{0}+\sum_{i=1}^{N_{\alpha}} S_{i} \alpha_{i}(t)+\sum_{i=1}^{N_{\alpha}} S_{i} \delta_{i}(t) .
\end{aligned}
$$

If only some of the scheduling variables has non-zero error part than (40) can be rewritten as:

$$
S(\bar{\alpha}(t))=S_{0}+\sum_{i=1}^{N_{\alpha}} S_{i} \alpha_{i}(t)+\sum_{j=1}^{N_{\delta}} S_{(i+j)} \delta_{j}(t),
$$

where $S_{(i+j)}$ are the matrices for which the error part is nonzero.

\section{REFERENCES}

[1] H. Kwakernaak and R. Sivan, Linear optimal control systems. WileyInterscience, 1972

[2] J. Willems, "Least squares stationary optimal control and the algebraic riccati equation," IEEE Transactions on Automatic Control, vol. 16, no. 6, pp. 621-634, Dec 1971.

[3] B. Molinari, "The time-invariant linear-quadratic optimal control problem," Automatica, vol. 13, no. 4, pp. 347 - 357, 1977.

[4] H. L. Trentelman and J. C. Willems, The Dissipation Inequality and the Algebraic Riccati Equation. Berlin, Heidelberg: Springer Berlin Heidelberg, 1991, pp. 197-242.
[5] V. Veselý, "Static output feedback controller design," Kybernetica, vol. 37, no. 2, pp. 205-221, 2001.

[6] D. Rosinová, V. Veselý, and V. Kučera, "A necessary and sufficien condition for static output feedback stabilizability of linear discrete-time systems," Kybernetika, vol. 39, no. 4, pp. 447-459, 2003.

[7] J. Engwerda and A. Weeren, "A result on output feedback linear quadratic control," Automatica, vol. 44, no. 1, pp. 265-271, 2008.

[8] S. Mukhopadhyay, "P.I.D. equivalent of optimal regulatror," Electronics Letters, vol. 14, no. 25, pp. 821-822, 1978.

[9] D. Rosinová and V. Veselý, "Robust static output feedback for discretetime systems - LMI approach," Periodica Polytechnica Ser. El. Eng. vol. 48, no. 3-4, pp. 151-163, 2004.

[10] V. Veselý, "Static output feedback robust controller design via LM approach," Journal of Electrical Eng., vol. 56, no. 1-2, pp. 3-8, 2005.

[11] — "Robust controller design for linear polytopic systems," Kyber netika, vol. 42, no. 1, pp. 95-110, 2006.

[12] D. Rosinová and V. Veselý, "Robust PID decentralized controller design using LMI," International Journal of Computers, Communications \& Control, vol. 2, no. 2, pp. 195-204, 2007.

[13] V. Veselý and D. Rosinová, "Robust PSD Controller Design," in Proceedings of the 18th International Conference on Process Control, M. Fikar and M. Kvasnica, Eds., Tatranská Lomnica, Slovakia, June 14-17 2011, p. 565570.

[14] — " "Robust PID-PSD Controller Design: BMI Approach," Asian Journal of Control, vol. 15, no. 2, pp. 469-478, 2013

[15] J. S. Shamma, Control of Linear Parameter Varying Systems with Applications. Springer, 2012, ch. An overview of LPV systems, pp. 3-26.

[16] V. Veselý and A. Ilka, "Gain-scheduled PID controller design," Journal of Process Control, vol. 23, no. 8, pp. 1141-1148, Sep. 2013.

[17] A. Ilka and V. Veselý, "Gain-Scheduled Controller Design: Variable Weighting Approach,” Journal of Electrical Engineering, vol. 65, no. 2, pp. 116-120, March-April 2014.

[18] V. Veselý and A. Ilka, "Design of robust gain-scheduled PI controllers," Journal of the Franklin Institute, vol. 352, no. 4, pp. 1476 - 1494, 2015.

[19] A. Ilka, V. Veselý, and T. McKelvey, "Robust Gain-Scheduled PSD Controller Design from Educational Perspective," in Preprints of the 11th IFAC Symposium on Advances in Control Education, Bratislava, Slovakia, June 1-3 2016, pp. 354-359.

[20] A. Ilka, I. Ottinger, T. Ludwig, M. Tárník, V. Veselý, E. Miklovicová, and J. Murgaš, "Robust Controller Design for T1DM Individualized Model: Gain-Scheduling Approach," International Review of Automatic Control (IREACO), vol. 8, no. 2, pp. 155-162, March 2015.

[21] V. Veselý and A. Ilka, "Unified Robust Gain-Scheduled and Switched Controller Design for Linear Continuous-Time Systems," Int. Review of Automatic Control (IREACO), vol. 8, no. 3, pp. 251-259, 2015.

[22] — "Generalized robust gain-scheduled PID controller design for affine LPV systems with polytopic uncertainty," Accepted to Systems and Control Letters, vol. 0, no. 0, pp. 0-0, 2017.

[23] P. Apkarian and P. Gahinet, "A convex characterization of gainscheduled $\mathrm{H} \infty$ controllers," IEEE Transactions on Automatic Control, vol. 40, no. 5, pp. 853-864, May 1995.

[24] P. Apkarian and H. D. Tuan, "Parameterized LMIs in Control Theory," SIAM Journal on Control and Optimization, vol. 38, no. 4, pp. 12411264, 2000.

[25] D. Petersson and J. Löfberg, "LPV H2-controller synthesis using nonlinear programming," in Proc. of the 18th IFAC World Congress, 2011.

[26] J. Bokor and G. Balas, "Linear Parameter Varying Systems: A Geometric Theory and Applications," IFAC Proceedings Volumes, vol. 38, no. 1, pp. $12-22,2005,16$ th IFAC World Congress.

[27] D. Henrion, J. Löfberg, M. Kočvara, and M. Stingl, "Solving polynomia static output feedback problems with PENBMI," in 44th IEEE Conference on Decision and Control, 2005 and 2005 European Control Conference. CDC-ECC '05., Dec 2005, pp. 7581-7586.

[28] J. Fiala, M. Kočvara, and M. Stingl, "Penlab: A matlab solver for nonlinear semidefinite optimization," October 2013, submitted to Mathematical Programming Computation. [Online]. Available: http://arxiv.org/pdf/1311.5240.pdf

[29] J. Löfberg, " YALMIP : A Toolbox for Modeling and Optimization in MATLAB," in Proc. of the CACSD Conference, Taipei, Taiwan, 2004

[30] H. Ichihara and M. Kawata, "Gain scheduling control of an arm-driven inverted pendulum based on sum of squares: Comparison with a SDRE method," IFAC Proceedings Volumes, vol. 44, no. 1, pp. 9613 - 9617 2011, 18th IFAC World Congress. 